\title{
The Gelling Ability of Some Diimidazolium Salts: Effect of Isomeric Substitution of the Cation and Anion
}

\author{
Francesca D'Anna, ${ }^{*[a]}$ Paola Vitale, ${ }^{[a]}$ Francesco Ferrante ${ }^{[b]}$ Salvatore Marullo, ${ }^{[a]}$ and \\ Renato Noto*[a]
}

\begin{abstract}
The gelling ability of some geminal imidazolium salts was investigated both in organic solvents and in water solution. Organic salts differing either in the cation or anion structure were taken into account. In particular, the effects on the gelphase formation of isomeric substitution on the cation or anion as well as of the use of mono- or dianions were evaluated. As far as the cation structure is concerned, isomeric cations, such as 3,3'-di-n-octyl-1,1'-(1,4-phenylenedimethylene)diimidazolium and 3,3'-di-n-octyl-1,1'-(1,3-phenylenedimethylene)diimidazolium, were used. On the other hand, in addition to the bromide anion, isomeric dianions, such as the 1,5- and 2,6-naphthalenedisulfonate anions, were also examined. After preliminary gelation tests, different factors affecting the obtained gel phases, such as the nature of the solvent, organoge-
\end{abstract}

lator concentrations, and action of external stimuli, were analyzed. The gel-phase formation was also studied as a function of time, by using resonance light scattering measurements. Ge morphologies were analyzed by scanning electron microscopy. To further support the understanding of the different behavior shown by the isomeric cations, some representative ion pairs were analyzed by DFT-based investigations. The collected data underline the significant role played by isomeric substitution of both cation and anion structures in determining the gelling capability of the investigated salts, as well as the properties of the gel phases. Finally, DFT investigations were helpful in the identification of the structural features affecting the self-assembly.

\section{Introduction}

The study of the properties and applications of soft materials originating from self-assembly processes is becoming an emerging research area. Liquid crystals, dendrimers, nanoparticles, and gels have attracted researchers' attention above all for the wide range of applications in which they can be involved. ${ }^{[1]}$ Among these different materials, gels have been the topic of many different reports in the literature. ${ }^{[2]}$ Indeed, after the pioneering work by Weiss on anthracene- and anthraquinone-based gels, ${ }^{[3]}$ a continuous growth in the number of papers concerning the gelling ability of polymers and low-molecular-weight gelators (LMWGs) as well as both neutral and charged species has occurred. ${ }^{[4]}$ The most widely accepted definition of gels is the one describing them as a three-dimensional fibrous network generated by self-assembly of gelator molecules in which the solvent is immobilized. This allows one to understand why gels have also been considered as "materi-

[a] Dr. F. D'Anna, P. Vitale, Dr. S. Marullo, Prof. R. Noto

Dipartimento Scienze e Tecnologie Biologiche, Chimiche e Farmaceutiche Università degli Studi di Palermo

Viale delle Scienze-Parco d'Orleans II, Ed. 17, 90128 Palermo (Italy)

Fax: (+ 39) 091596825

E-mail:francesca.danna@unipa.it

renato.noto@unipa.it

[b] Dr. F. Ferrante

Dipartimento di Fisica e Chimica, Università degli Studi di Palermo Viale delle Scienze-Parco d'Orleans II, Ed. 17, 90128 Palermo (Italy)

Supporting information for this article is available on the WWW under http://dx.doi.org/10.1002/cplu.201300016. als at the interface between complex fluids and the phase-separated state of matter". ${ }^{[5]}$

Independently of the applicative implications related to the obtaining of a gel phase, one of the most attractive aspects of this kind of investigation is to try to understand how to conjugate solvent and gelator properties to improve the features of the obtained gels. Serendipity has been frequently considered as a crucial element in gel phase discovery. The only certainty derives from the knowledge that, as a result of a self-assembly process, gel formation is driven by the action of feeble but cooperative supramolecular forces. Consequently, the presence in gelator molecules of some structural features favoring or disfavoring the establishment of such kinds of interactions could represent the starting point to design the target molecule. The identification of factors that give rise to gel formation could be important to compare the effects that small changes in gelator structure induce in the gel properties. However, notwithstanding the amount of research activity developed in this field, this knowledge is still elusive.

Among LMWGs, in the last few years a certain amount of attention has been devoted to organic salts. Partly, this interest is because of the presence in these tectons of reasonably strong directional hydrogen-bond sites. As recently stated by Dastidar, this structural feature should favor 1D fiber formation that subsequently might lead to self-assembled fibrillar networks. ${ }^{[6]}$ From an applicative point of view, conductive gels might be quite interesting as highly polar reaction media. Moreover, recently, a hypothesis of their application in dye-sen- 
sitized solar cells has been put forward, which avoids the use of more corrosive inorganic salts. ${ }^{[7]}$ In this light the gelation ability of ammonium, ${ }^{[7 \mathrm{~b}, \mathrm{c}]}$ imidazolium, ${ }^{[8]}$ benzotriazolium, ${ }^{[9]}$ and benzylammonium ${ }^{[10]}$ salts has been tested.

In this context, we have recently reported data on the gelling ability of some geminal diimidazolium salts, thus evidencing how the properties of the obtained soft materials depend on the length of the alkyl chain on the imidazolium ion and the nature of the anion. ${ }^{[11]}$ To better identify factors affecting the gelling behavior of such kinds of salts and bearing in mind the influence of size and shape of tectons in the self-assembly processes, we thought it could be interesting to study how the different natures of anion or cation could affect the gelphase formation. In this light, we examined two cations different in shape, namely $3,3^{\prime}$-di-n-octyl-1, $1^{\prime}-(1,4$-phenylenedimethylene)diimidazolium ([p-Xyl-(oim) $\left.{ }_{2}{ }^{2+}\right]$ ) and 3,3'-di-n-octyl-1,1'(1,3-phenylenedimethylene)diimidazolium $\quad\left(\left[m-X y l-(o i m)_{2}{ }^{2+}\right]\right)$. Indeed, the different isomeric substitution might induce changes in gelation ability and consequently different properties of the obtained materials. To this purpose, the role played by the isomeric substitution has proved to be important in determining the properties and structural features of some geminal diimidazolium ionic liquids. ${ }^{[12]}$

As far as anions are concerned, we used the bromide anion for its high coordination ability and two isomeric dianions. The interest in dianions came from the possibility of having two charged heads on the same spacer. This could induce significant changes in the cation-anion interactions, with respect to the ones detected in the presence of the bromide anion, and on the whole it could affect the three-dimensional network formation needed to give the gel phase. In particular, we took into account aromatic dianions, such as the 1,5- and 2,6-naphthalenedisulfonate anions ([1,5-NDS] and [2,6-NDS]). Indeed, the presence of a rigid aromatic spacer able to favor the establishment of $\pi-\pi$ cation-anion interactions could be a further structural element determining the packing of molecules within the fibrillar networks. Also in this case, we used structural isomers to evaluate how gel properties were affected by a different electronic distribution and hydrogen-bond direction (Scheme 1).

After preliminary gelation tests, all the obtained soft materials were investigated for their possible thixotropic and sonotropic behavior, which could have important implications from an applicative point of view. Indeed, these phenomena are typical of molecular assemblies that are destroyed under mechanical stimuli or ultrasound irradiation but repair in a self-assembling manner. In particular, in the case of LMWGs the study of these processes is interesting as a consequence of their dynamic nature. ${ }^{[13]}$ Furthermore, self-healing is a common process in biological systems, ${ }^{[14]}$ and artificial systems able to selfheal could be a useful tool to gain a better understanding of this process. The obtained materials play a crucial role as functional soft materials. ${ }^{[15]}$

The thermodynamic stability of the obtained gels was studied as a function of both the nature of the solvent used and organogelator concentration. To acquire information about the role played by the solvent in molecular gelation, we deter-

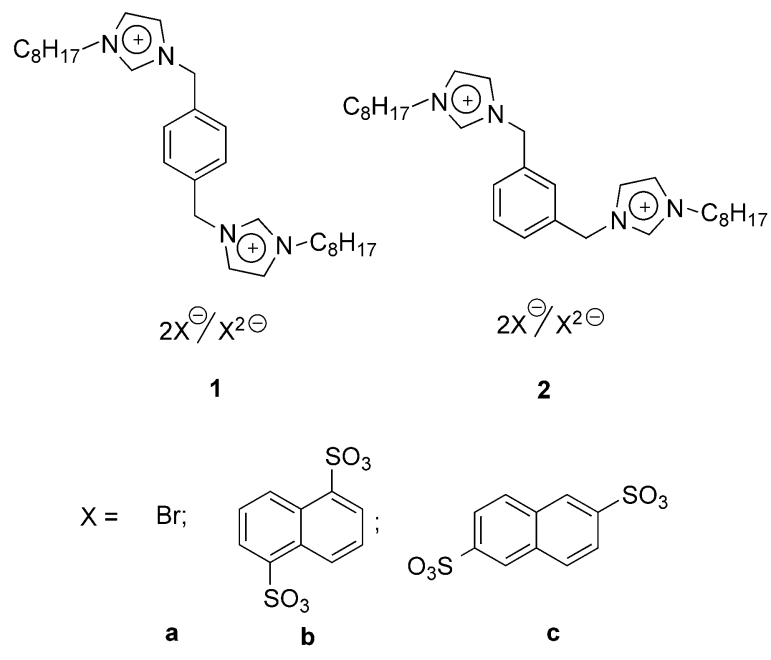

Scheme 1. Structure of the organic salts used.

mined dissolution parameters from the variable-temperature solubilities by using the van't Hoff equation. ${ }^{[16]}$ In all cases in which a gel phase was obtained, we tried also to correlate the gel melting temperature $\left(T_{\text {gel }}\right)$ with solvent parameters. The aggregation process was investigated by using resonance light scattering (RLS) measurements. Gel morphologies were analyzed by scanning electron microscopy (SEM). Finally, we performed density functional theory (DFT)-based investigations on the [p-Xyl-(oim) $\left.)_{2}\right][1,5-N D S]$ (1 b), [p-Xyl-(oim) 2$][2,6-N D S]$ (1 c), and [m-Xyl-(oim) $\left.)_{2}\right]$ 1,5-NDS] (2 b) ion pairs to obtain information on the relation between the anion-cation interaction geometries and the gelling capability of these compounds.

\section{Results and Discussion}

\section{Gelation tests}

Gelation tests were performed with solvents of different polarity and hydrogen-bond donor abilities. In particular, both protic and aprotic polar solvents were taken into account. In all cases in which gel formation was observed the critical gelation concentration (CGC; i.e., the lowest organogelator concentration inducing gel formation) and the $T_{\text {gel }}$ values (i.e., the melting temperature of the gel) were determined. Data collected in the presence of all organic salts as a function of the solvents used are reported in Table 1.

Analysis of the data in Table 1 evidences the important role played by both cation and anion structure in favoring gel formation. Indeed, independently of cation structure, the bromides ( $\mathbf{1} \mathbf{a}$ and $\mathbf{2}$ a) showed a lower ability to induce gel formation. Indeed, the formation of a stable opaque gel was detected only for $\mathbf{1}$ a in acetonitrile solution. In general, bromides had good solubility in protic polar solvents and, in the analyzed concentration range, they were insoluble in aromatic solvents. This result agrees with the one recently obtained by $\mathrm{us}^{[11]}$ through studying the gelling behavior of bromides bearing decyl and dodecyl alkyl chains on the cation structure, and 


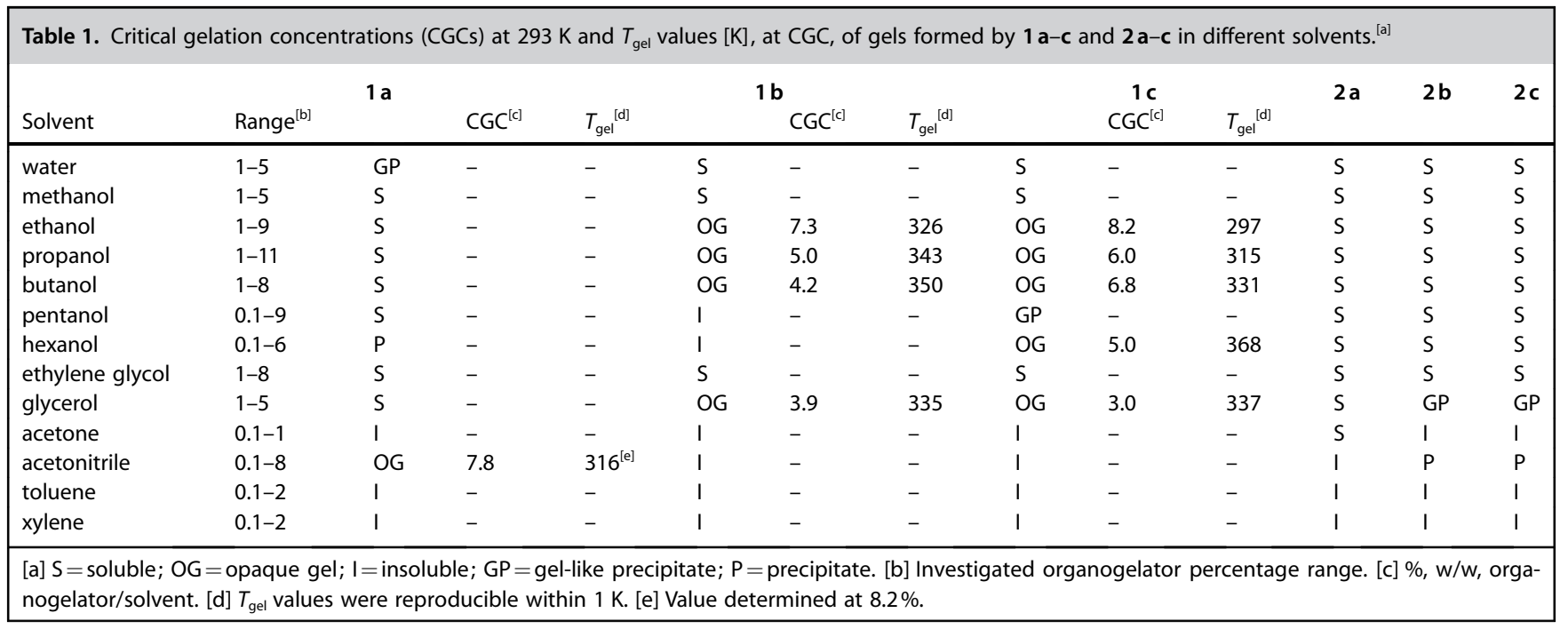

seems to indicate a low efficiency of the bromide anion in promoting the self-assembly process.

On the other hand, taking into account naphthalenedisulfonate salts, the gelation ability was observed only in the presence of the $\left[p\right.$-Xyl-(oim) $\left.{ }_{2}{ }^{2+}\right]$ cation. As for the salts based on $\left[\mathrm{m}\right.$-Xyl-(oim) $\left.{ }_{2}{ }^{2+}\right](\mathbf{2} \mathbf{b}$ and $\mathbf{2} \mathbf{c})$, they showed good solubility in all protic polar solvents but they were insoluble in aprotic polar and aromatic solvents.

Perusal of Table 1 shows that $\mathbf{1} \mathbf{b}$ and $\mathbf{1} \mathbf{c}$ were able to behave only as organogelators. In particular, they were insoluble in aprotic polar solvents, such as acetonitrile and acetone, and in aromatic solvents, such as toluene and xylene. By contrast, they gave clear solutions even at room temperature in polar protic solvents, such as water or methanol.

The first evidence about gelation ability was obtained on dissolving the above organic salts in ethanol. In both cases opaque gels were obtained. They showed significantly different stabilities as accounted for by their $T_{\text {gel }}$ values $\left(T_{\text {gel }}=326\right.$ and $297 \mathrm{~K}$ for $\mathbf{1} \mathbf{b}$ and $\mathbf{1 ~ C}$, respectively). Moreover, both organogelators gave rise to stable, thermoreversible, opaque gels in propanol, butanol, and glycerol solutions. In the case of $\mathbf{1} \mathbf{c}$, the formation of a gel phase was also observed in hexanol solution.

The macroscopic behavior of the gels was analyzed initially by monitoring the transition from an immobile to a mobile self-assembly state by using the "tube inversion test" (Figure 1). This simple methodology is widely used to assess the gel formation of low-molecular-weight organogelator. ${ }^{[17]}$ In these conditions, our gels remained stable for almost three months at room temperature.

Analysis of the data reported in Table 1 allows one to highlight some differences between the gelation abilities of the two tested compounds. In general, with the exception of glycerol, the CGC was lower for $\mathbf{1} \mathbf{b}$ than for $\mathbf{1}$ c. Furthermore, for the same organic solvent, gels formed by $\mathbf{1} \mathbf{b}$ were more stable than those formed by $1 \mathrm{c}$, as accounted for by the $T_{\text {gel }}$ values.

These results seem to indicate that the different substitution pattern on the naphthalene core of the anion is able to induce (a)

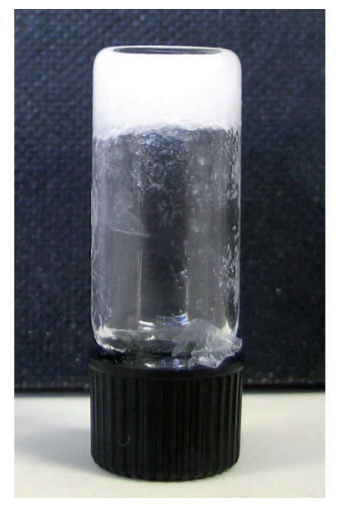

(b)

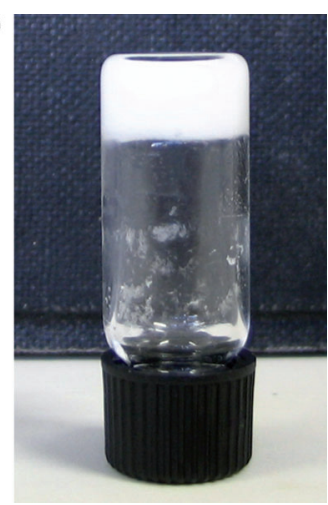

Figure 1. Opaque gels formed by (a) 1 b $(5 \%, w / w)$ and (b) 1 c $(6 \%, w / w)$ in propanol.

significant differences in the self-assembly process. This is also confirmed by the differences in gelation ability shown by $\mathbf{1} \mathbf{~ b}$ and $1 \mathrm{c}$ with the nature of the solvent used. In general, the nature of the alcohol also affects the stability of the gel material. Indeed, the stability increases and the CGC decreases for more hydrophobic alcohols. No significant differences in gel material stabilities were detected in glycerol.

To gain a deeper understanding of the results collected by carrying out gelation tests, we determined dissolution enthalpy and entropy values for some of the solvent-gelator systems analyzed in this work. Furthermore, computational investigations were also performed on some selected ion pairs to identify how the structural features and the geometry or strength of cation-anion interactions affected the gelling ability.

\section{Dissolution enthalpies and entropies}

For correlation between the gelator structure and gelling ability, two different theories have been reported in the literature. The first, reported by Day and co-workers, ${ }^{[18]}$ correlates the gelation ability with the propensity to form 1D hydrogenbonded networks. A different approach reported by Shinkai 
and co-workers, and recently reasserted by McNeil and coworkers, ${ }^{[19]}$ makes a clear distinction between gelators and nongelators on the grounds of the dissolution enthalpy and entropy values, and ascribes the highest gelation abilities to species having the highest dissolution enthalpy. This latter approach has also been used by Smith and co-workers, ${ }^{[20]}$ who outlined a sort of correlation between dissolution parameters and CGC values.

On the grounds of all the above information, we measured the equilibrium solubilities by means of UV/Vis measurements and the van't Hoff equation, ${ }^{[16]}$ in the temperature range $283-$ $413 \mathrm{~K}$. In particular, in the case of $1 \mathbf{b}$ and $1 \mathrm{c}$, with the exception of toluene and xylene, we determined the dissolution parameters in all the tested solvents. For toluene and xylene, the low solubilities of gelators in these solvents and the superimposition of the UV/Vis band of gelator and solvent did not allow good reproducibility of the collected data. Furthermore, for a comparison between gelators and nongelators, we took acetonitrile as reference solvent system and determined the dissolution parameters for all organic salts. Dissolution enthalpies and entropies are reported in Table S1 of the Supporting Information. (In Table S2, $\lambda_{\max }$ and $\varepsilon$ values used to determine dissolution enthalpies and entropies are reported. In Table S3 the dissolved mole fractions of gelator as a function of the temperature values are reported.) For better visualization of the collected data, they are presented in Figure 2.

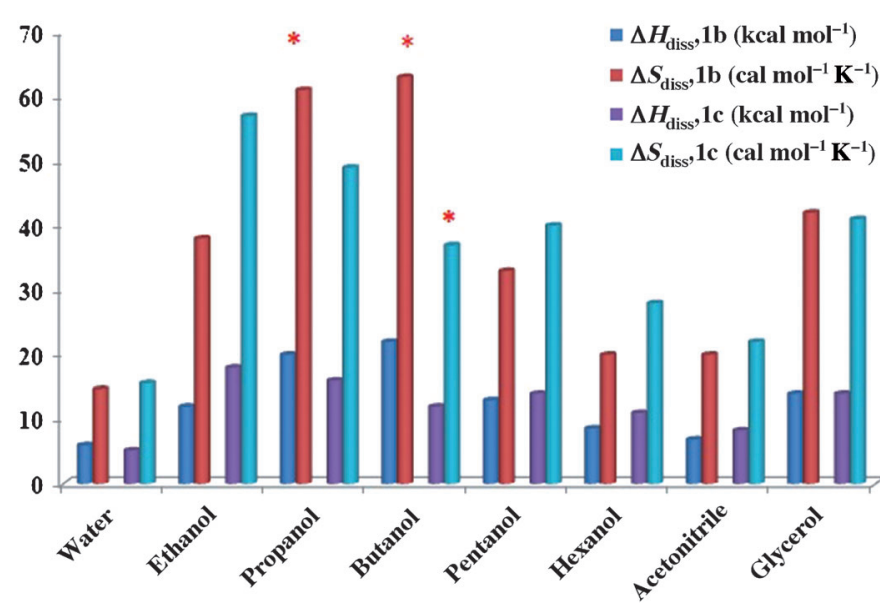

Figure 2. Enthalpies and entropies of the dissolution process for gelators $\mathbf{1} \mathbf{b}$ and $\mathbf{1} \mathbf{c}$ in different solvents (gelator-solvent systems that give rise to gel phases are indicated with a star).

Perusal of the data reported in Table S1 and Figure 2 allows us to make some useful considerations. Firstly, taking into account data relevant to $\mathbf{1} \mathbf{b}$ and $\mathbf{1} \mathbf{c}$, wider ranges in the thermodynamic parameters of dissolution were detected for $\mathbf{1} \mathbf{b}$ than for $1 \mathrm{c}$. Indeed, enthalpy values ranged from 5.9 to $22 \mathrm{kcal}$ $\mathrm{mol}^{-1}$ for $1 \mathbf{b}$ and from 5.2 to $18 \mathrm{kcal} \mathrm{mol}^{-1}$ for $1 \mathrm{c}$. Analogously, the entropy values ranged from 15 to $63 \mathrm{cal} \mathrm{mol}^{-1} \mathrm{~K}^{-1}$ for $1 \mathbf{b}$ and from 16 to $49 \mathrm{cal} \mathrm{mol}^{-1} \mathrm{~K}^{-1}$ for $1 \mathrm{c}$.

In particular, for the same gelator, the dissolution parameters increase on going from a solute-solvent system behaving as a nongelling system to one that gives rise to gel-phase formation (compare entries 2-4, 8 and 1, 5-7 of Table S1 for $\mathbf{1} \mathbf{b}$ and entries $2-4,6,8$ and 1, 5, 7 of Table $S 1$ for $1 \mathrm{c}$ ). This result seems to indicate, according to previous reports in the literature, ${ }^{[19 b, c]}$ that gel-phase formation is favored by the occurrence of weaker solute-solvent interactions. Data collected by using $\mathbf{1 b}$ and $\mathbf{1 c}$ allow further comparisons. The first, in which the solvent is the same, should allow evaluation of the effect derived from the isomeric substitution on the naphthalene core. The second, in which the gelator is the same, should give information about the solvent effect on the gelator dissolution process.

In the first case, data collected in alcohol solutions for $\mathbf{1} \mathbf{b}$ and $1 \mathrm{c}$ do not delineate a clear trend. Indeed, the dissolution enthalpies were equal in glycerol solution, lower for $\mathbf{1} \mathbf{b}$ than for $\mathbf{1} \mathbf{c}$ in ethanol, and higher for $\mathbf{1 b}$ than for $\mathbf{1} \mathbf{c}$ in propanol and butanol.

On the other hand, for the same organic cation, the isomeric anions seem to induce a different sensitivity of the dissolution process to the solvent properties. In particular, as for data collected in alcohol solution, in the case of $\mathbf{1} \mathbf{b}$ the dissolution enthalpy increases on going from ethanol to butanol, with the increase in the alkyl chain length and consequently with the solvent polarity decrease $\left(E_{T(30)}=51.9,50.7\right.$, and $50.2 \mathrm{kcal} \mathrm{mol}^{-1}$ for ethanol, propanol, and butanol, respectively). ${ }^{[21]}$ An opposite trend was detected in the case of $1 \mathrm{c}$. In this case the solvent polarity decrease seems to reduce intermolecular interactions between gelator molecules, thus favoring the occurrence of a dissolution process at lower enthalpy values. On the grounds of the above results and bearing in mind data previously reported in the literature, ${ }^{[20]}$ we searched for a correlation between the dissolution enthalpies and the CGC values. However, bad correlations were obtained in both cases, which probably indicated, according to previous reports, ${ }^{[19 c]}$ that in our case different factors besides the dissolution process contribute to determining the CGC values.

Finally, as previously stated, we collected dissolution parameters for all the salts in acetonitrile solution. Indeed, in this solvent all salts showed enough solubility for us to carry out UV/ Vis measurements, thus allowing determination of the dissolution parameters. All of these latter results could represent an experimental contribution to the understanding of the reason why the $\left[m\right.$-Xyl-(oim) $\left.{ }_{2}{ }^{2+}\right]$-based salts did not show gelling abilities. Analysis of data collected in acetonitrile solution reveals how the dissolution parameters are significantly affected by the cation structure, as accounted for by the decrease in both enthalpy and entropy values on going from the $\left[p\right.$-Xyl-(oim) $\left.{ }_{2}{ }^{2+}\right]$ to $\left[m\right.$-Xyl-(oim) $\left.{ }_{2}{ }^{2+}\right]$ cation. In general terms, the above decrease was more significant for naphthalenedisulfonate than for bromide salts.

As for the anion effect, for the same cation a significant change in dissolution parameters was detected on going from bromide to naphthalenedisulfonate salts, which indicated the occurrence of very different solute-solvent interactions in the presence of aromatic dianions. However, less significant changes were detected as a consequence of the different isomeric substitution. On the grounds of all the above results, the 
lower ability of $\left[m-\mathrm{Xyl}\right.$-(oim) $\left.{ }_{2}{ }^{2+}\right]$ salts to give rise to gel-phase formation could be ascribed to the more pronounced ability of this cation to give strong solute-solvent interactions.

\section{Computational investigations}

The computational investigations reported herein aimed to find a correlation between the structural characteristics of cations 1 and 2 and the geometry/strength of their interaction with the anion, and hence to give some explanation of their different behavior with respect to gelation. To obtain such information the ion pairs $\mathbf{1} \mathbf{b}, \mathbf{1} \mathbf{c}$, and $\mathbf{2} \mathbf{b}$, as well as the systems cation-anion-cation $(\mathbf{1})_{2} \mathbf{b},(\mathbf{1})_{2} \mathbf{c}$, and $(2)_{2} \mathbf{b}$, were subjected to full geometry optimization by means of DFT approaches. By choosing the starting geometries it was assumed that, in the ion pairs, the naphthalenedisulfonate anion interacts simultaneously with the two positively charged imidazolium rings of the cation.

In the ion pairs $\mathbf{1} \mathbf{b}, \mathbf{1} \mathbf{c}$, and $\mathbf{2} \mathbf{b}$ (see Figure 3 ), the leading interactions occur, as expected, between the sulfonate oxygen atoms and the imidazolium $\mathrm{H} 2$ atom. Incidentally, these seem to be strengthened by the interactions of the same oxygen atoms with the hydrogen atoms of the benzyl group and those in the $\alpha$ position of the octyl chains, which are at distances (2.12-2.32 $\AA$ ) and orientations consistent with the formation of weak hydrogen bonds. Indeed, these hydrogen atoms are characterized by relatively high partial positive charges, as calculated by fitting the atomic charges to the molecular electrostatic potential method. To achieve more efficient electro- static interactions the cation adopts, in all the ion pairs investigated a conformation that is not completely extended as it occurs in the most stable conformation of the isolated $\mathbf{1}$ and $\mathbf{2}$ cation species.

The total interaction energies in the three ion pairs have been calculated as $\mathbf{2 b}\left(277 \mathrm{kcal} \mathrm{mol}^{-1}\right)>\mathbf{1}$ c $\left(272 \mathrm{kcal} \mathrm{mol}^{-1}\right)>$ 1 b $\left(269 \mathrm{kcal} \mathrm{mol}^{-1}\right)$. The most substantial difference between the three ion pairs is with regard to the mutual position of the cation phenyl ring and the anion naphthalene ring. In $\mathbf{1} \mathbf{b}$ and $1 \mathrm{c}$ there is no structural evidence that can lead one to suppose the occurrence of face-to-face $\pi$ interactions, whereas in $\mathbf{2} \mathbf{b}$ the phenyl and naphthyl moieties lie in planes almost parallel to each other, with a graphite-like arrangement and corresponding atom-atom minimum and maximum distances of 3.2 and $3.9 \AA$, respectively. This would mean that the whole of the electrostatic interaction in $\mathbf{2} \mathbf{b}$ allows the adoption of a geometry that promotes the arising of efficient face-to-face $\pi$ interactions.

To investigate further the geometry of the interactions between the di-n-octyl-phenylenedimethylene-diimidazolium cation and the naphthalenedisulfonate anion, the cationanion-cation systems $(1)_{2} \mathbf{b},(1)_{2} \mathbf{c}$, and $(2)_{2} \mathbf{b}$ were considered. In the optimized geometry of these species each sulfonate group of the anion interacts simultaneously through hydrogen bonds with two imidazolium rings belonging to different cations. As shown in Figure 3 , in (2) ${ }_{2} \mathbf{b}$ the anion is sandwiched between two cationic species and the naphthalene ring is in a plane parallel to the phenyl rings. This geometry concurs to the maintaining of efficient $\pi$ interactions in this system. On the other hand, in $(1)_{2} b$ and $(1)_{2} \mathrm{C}$, again the interaction geometry seems to hamper the occurrence of face-to-face interactions. Further, the interaction geometry in (2) ${ }_{2}$ b allows the octyl chains of the two cations to interact with each other and to form a sort of cage around the anion. The same interactions are also present in $(1)_{2} \mathrm{~b}$ and $(1)_{2} \mathrm{c}$ to some extent, but they seem to be restricted to the terminal portions of the octyl chains, so relieving the anion caging effect.

Joint analysis of the results derived from dissolution parameter determination and computational investigation gives general insights into the different effects of isomeric substitution on the cations in the gelation process. Indeed, as frequently stated in the literature, a necessary and sufficient condition to have a gel phase is the formation of one-dimensional aggregates that join to form a three-dimensional net-

Figure 3. Fully optimized DFT geometries of the ion pairs $\mathbf{1} \mathbf{b}, \mathbf{1} \mathbf{c}$, and $\mathbf{2} \mathbf{b}$ ( $\mathbf{a}-\mathrm{c}$, respectively) and of the same ion pairs interacting with a second cation, $(\mathbf{1})_{2} \mathbf{b},(\mathbf{1})_{2} \mathbf{c}$, and $(\mathbf{2})_{2} \mathbf{b}(\mathrm{d}-\mathrm{f}$, respectively). 
work. The picture obtained from the computational results seems to depict $\mathbf{2} \mathbf{b}$ as a species in which stronger interactions can be realized in the ion pair with respect to $\mathbf{1} \mathbf{b}$. The coulombic interaction between the cation and the anion in $\mathbf{2} \mathbf{b}$ seems to be reinforced by face-to-face $\pi$ stacking and weak interactions between the alkyl chains, both allowed by the interaction geometry; this could also result in closed cages or oligomeric systems formed by weakly interacting units. Most likely, $\mathbf{2} \mathbf{b}$ is not very willing to reorganize to give efficient intermolecular interactions and the ion pair, or greater aggregates, should interact better with solvent molecules, as indicated by thermodynamic dissolution parameters, so resulting in the lack of ability to give the gel network.

The obtaining of a gel is clearly addressed to its use. However, to choose a specific application for a soft material derived from a self-assembly process of LMWGs, knowledge of all the factors that can affect its thermal stability and morphology as well as the mechanism and rate of the gelation process are necessary. Furthermore, for some fields of application, a given gel phase could be subjected to the action of external stimuli. In this light, it could be interesting to evaluate not only the thermoreversibility of the soft material, but also its response to a mechanical stress or ultrasound irradiation.

Bearing in mind all the above considerations, and with the future aim to use the gel phases formed by $\mathbf{1 b}$ and $\mathbf{1 c}$ in the study of molecular recognition processes or as organized reaction media, we evaluated the solvent effect on the thermal stability and morphology of the obtained gel phases, as well as on the ability of these materials to perform self-healing processes.

\section{Solvent effect on gel-phase formation}

Firstly, we tried to correlate $T_{\text {gel }}$ values corresponding to gel phases formed by $\mathbf{1} \mathbf{b}$ and $\mathbf{1} \mathbf{c}$ to solvent properties such as polarity. Different attempts tried to quantify the solvent effect on gelation processes and different indicators have been used, such as polarity, Kamlet-Taft, or Hildebrand solubility parameters. ${ }^{[22]}$

$T_{\text {gel }}$ values as a function of the different nature of the organogelator and solvent are reported in Table S4 together with solvent parameters. Perusal of the data seems to indicate that the thermal stability of the gel phases decreases with solvent polarity, as accounted for by correlation between $T_{\text {gel }}$ values and solvent polarity parameters such as $\pi^{*}, E_{\mathrm{T}(30)}$, or dielectric constant values. Differently, no regular trend was detected that takes into account the hydrogen-bond donor or acceptor ability. We are aware of the fact that this could also be a consequence of the narrow range of the above parameters.

The decrease in $T_{\text {gel }}$ values with the increase of solvent polarity could indicate that, for our gelators, a more polar solvent disfavors the gelation process as a consequence of the competing interactions with gelator molecules, and this finding agrees with the one previously reported by Tritt-Goc and coworkers. ${ }^{[22 c]}$ The result now obtained is completely different from the one we found recently by using as organogelators geminal imidazolium salts having monoanions such as bro- mide and tetrafluoroborate. ${ }^{[11]}$ Probably, this different behavior agrees with the statement frequently reported in the literature about the possibility that the obtaining of such a correlation is strictly dependent on gel microstructure, which changes frequently with gelator and solvent structure.

\section{Thixotropic and sonotropic behavior}

The obtained gels were investigated for their ability to respond to external stimuli. Although the reversibility of the self-assembly process induced by thermal stimuli is generally reported for LMWGs, thixotropy and sonotropy are exhibited only by a few gelators.

For comparison of the obtained gels, they were tested at $7 \%(\mathrm{w} / \mathrm{w})$ and the data collected are reported in Table S5. Analysis of the results shows that our gelators exhibited very different behaviors. Compound $\mathbf{1} \mathbf{b}$ gave rise to a thixotropic gel in glycerol solution, whereas gels formed by $1 \mathrm{c}$ showed a more distinct stimuli-responsive behavior. In particular, thixotropy was detected in both propanol and glycerol solutions and this latter gel was also able to behave as a sonotropic phase. To obtain information about the strength of supramolecular interactions in thermal and both re-formed thixotropic and sonotropic gel phases, we compared $T_{\text {gel }}$ values. In general, the action of external stimuli seems to weaken the strength of supramolecular interactions. In particular, for two of the analyzed cases ( $\mathbf{1} \mathbf{b}$ in glycerol and $\mathbf{1} \mathbf{c}$ in propanol solution) the action of magnetic stirring gave rise to gel phases softer than the corresponding thermotropic ones.

No apparent change in the thermodynamic stability was detected after the ultrasound irradiation of the gel phase formed by $1 \mathrm{c}$ in glycerol solution. Interestingly, the thixotropic phase re-formed by $1 \mathrm{c}$ in glycerol solution was more stable than the corresponding thermotropic one. On the whole, the data collected indicate that the self-healing process is affected significantly by the nature of both solvent and anion. In general, the self-healing process is favored in the presence of the anion less able to take part in directional interactions, as accounted for by data corresponding to $\mathbf{1 b}$ and $\mathbf{1 c}$ in glycerol solution. On the other hand, for the same anion (1 $\mathrm{c}$ in propanol and glycerol solutions) magnetic stirring seems to favor fiber entanglement in the presence of solvents, such as glycerol, that have a higher hydrogen-bond donor ability and might compete with tectons in fibrous network formation. Furthermore, in the only case in which the self-healing process was a result of ultrasound irradiation ( $\mathbf{1} \mathrm{c}$ in glycerol solution), the action of the external stimulus seems to weaken the supramolecular interactions. This result agrees with that reported recently by $\mathrm{Yi}$ et al. upon studying the sonication effects on gel phases formed by peptide-based LMWGs. ${ }^{[13 e]}$

The information obtained about the general behavior of gel phases formed by $\mathbf{1} \mathbf{b}$ and $\mathbf{1} \mathbf{c}$ prompted us to choose some of them as reference systems to gain a deeper understanding of the effects derived from the different nature of the anion. To pursue this goal, we analyzed as reference systems the gel phases formed by $\mathbf{1} \mathbf{b}$ and $\mathbf{1} \mathbf{c}$ in propanol solution and investigated their thermodynamic stability, gel-phase formation as 
a function of time, as well as their morphology. In some cases, to obtain information about the solvent effect, collected data for butanol gel phases were also taken into account.

\section{Thermodynamic stability}

The stability of the gels formed in propanol was analyzed as a function of the organogelator concentration. Figure 4 shows
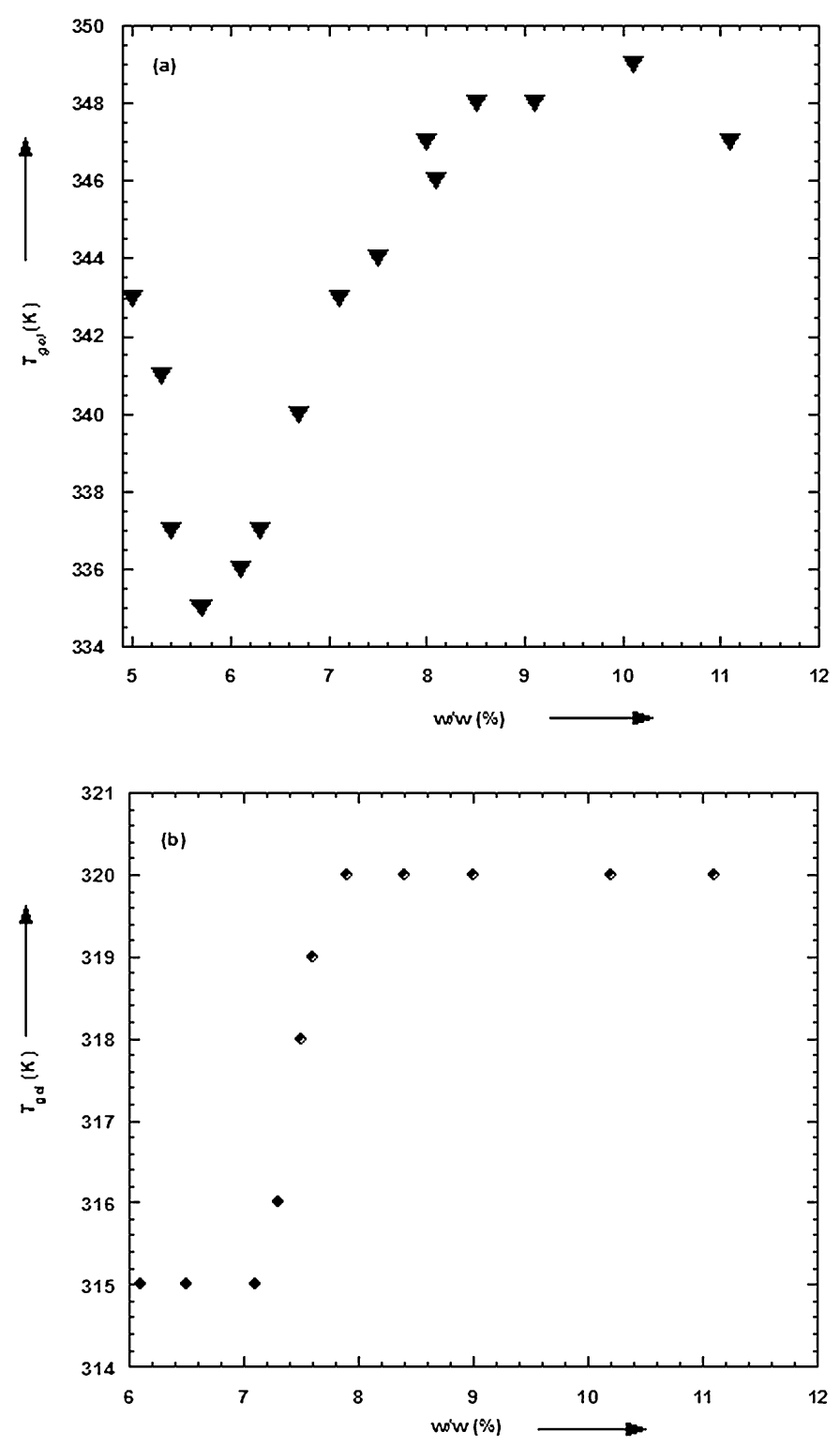

Figure 4. Plots of $T_{\text {gel }}[\mathrm{K}]$ versus organogelator concentration in propanol corresponding to: (a) $\mathbf{1} \mathbf{b}$ and (b) $\mathbf{1} \mathbf{c}\left(T_{\text {gel }}\right.$ values were reproducible within $1 \mathrm{~K})$.

plots of $T_{\text {gel }}$ as a function of $\mathbf{1} \mathbf{b}$ and $\mathbf{1 c}$ concentration ( $T_{\text {gel }}$ values as a function of the organogelator concentration are also reported in Table S6). In general, $T_{\text {gel }}$ values change in a wider range for $\mathbf{1} \mathbf{b}$ (from 335 to $349 \mathrm{~K}$ ) than for $\mathbf{1} \mathbf{c}$ (from 315 to $320 \mathrm{~K}$ ). For $1 \mathbf{b}$ (see Figure $4 \mathrm{a}$ ), the lowest $T_{\text {gel }}$ value, de- tected in the concentration range from 5 to $6 \%(w / w)$, was chosen as the minimum value. The trend obtained in the case of $\mathbf{1} \mathbf{b}$ is quite uncommon. In general, such a kind of plot previously reported in the literature shows a trend similar to that obtained for $\mathbf{1} \mathbf{c}$. In an attempt to explain the data collected for $\mathbf{1} \mathbf{b}$, we supposed that in the analyzed concentration range a reorganization of the gel-phase three-dimensional network occurred, and this hypothesis was well supported by changes detected in the gel-phase morphology with the organogelator concentration (see below).

We can interpret roughly the above different trends in $T_{\text {gel }}$ values of $\mathbf{1} \mathbf{b}$ and $\mathbf{1} \mathbf{c}$ by taking into account the differences in electronic properties of the anions used. Firstly, the isomeric substitution in the anions should induce a different directionality to cation-anion hydrogen bonds and probably this could affect the strength of the above interactions. Furthermore, the anions also differ in the quadrupole moment value. The $Q_{x x}$ component of the traceless quadrupole moment of [1,5NDS] and [2,6-NDS] anions was calculated as equal to -88 and $-132 \mathrm{DA}$, respectively, by using the second-order MoellerPlesset perturbative method with the cc-pvdz basis set. Then, on the grounds of these considerations, we can suppose that the observed behavior for two gelators was the result of the different weight of the above contributions.

To further analyze the stability of the obtained materials we determined, by using the equation previously reported, ${ }^{[23]}$ the enthalpy variation for the gel-sol transition $\left(\Delta H_{f}\right)$ in the two cases (plots of $\ln C$ versus $1 / T$ are reported in Figure $S 1$ of the Supporting Information). In particular, the attempt to calculate $\Delta H_{\mathrm{f}}$ values by considering all experimental data gave rise to bad correlations. Consequently, we took into account the range in which an increase of $T_{\text {gel }}$ with the organogelator concentration was detected. Analysis of $\Delta H_{\mathrm{f}}$ values reveals that the gel-sol transition is affected significantly by the nature of the organogelator. The enthalpy variation decreased from 6.7 to $4.3 \mathrm{kcal} \mathrm{mol}^{-1}$ on going from $\mathbf{1} \mathbf{b}$ to $\mathbf{1} \mathbf{c}$, once more outlining a larger stability for the soft materials formed by $[p$-Xyl(oim) $\left.)_{2}\right][1,5-\mathrm{NDS}]$, as a consequence of a higher relevance of directional interactions.

\section{Kinetic study of organogel formation}

As stated above, the formation of the self-assembled fibrillar network was also studied as a function of time. To investigate the nucleation and growth of the fibrous network, we used RLS measurements. This technique is largely employed to study the aggregation processes of very different systems. ${ }^{[24]}$ It is well known that changes in the RLS intensity can be ascribed to changes in the size, shape, and concentration of the aggregates. ${ }^{[25]}$ Thus, in our case it allowed us to obtain information about both the rate of the aggregation process and the size of the aggregates derived from fiber-fiber interactions in the gel network. To obtain information about the effect of the organogelator concentration on the aggregate size, studies as a function of concentration were also performed. Figure 5 shows plots of RLS intensity as a function of time for both organogelators in propanol solution at room temperature. 

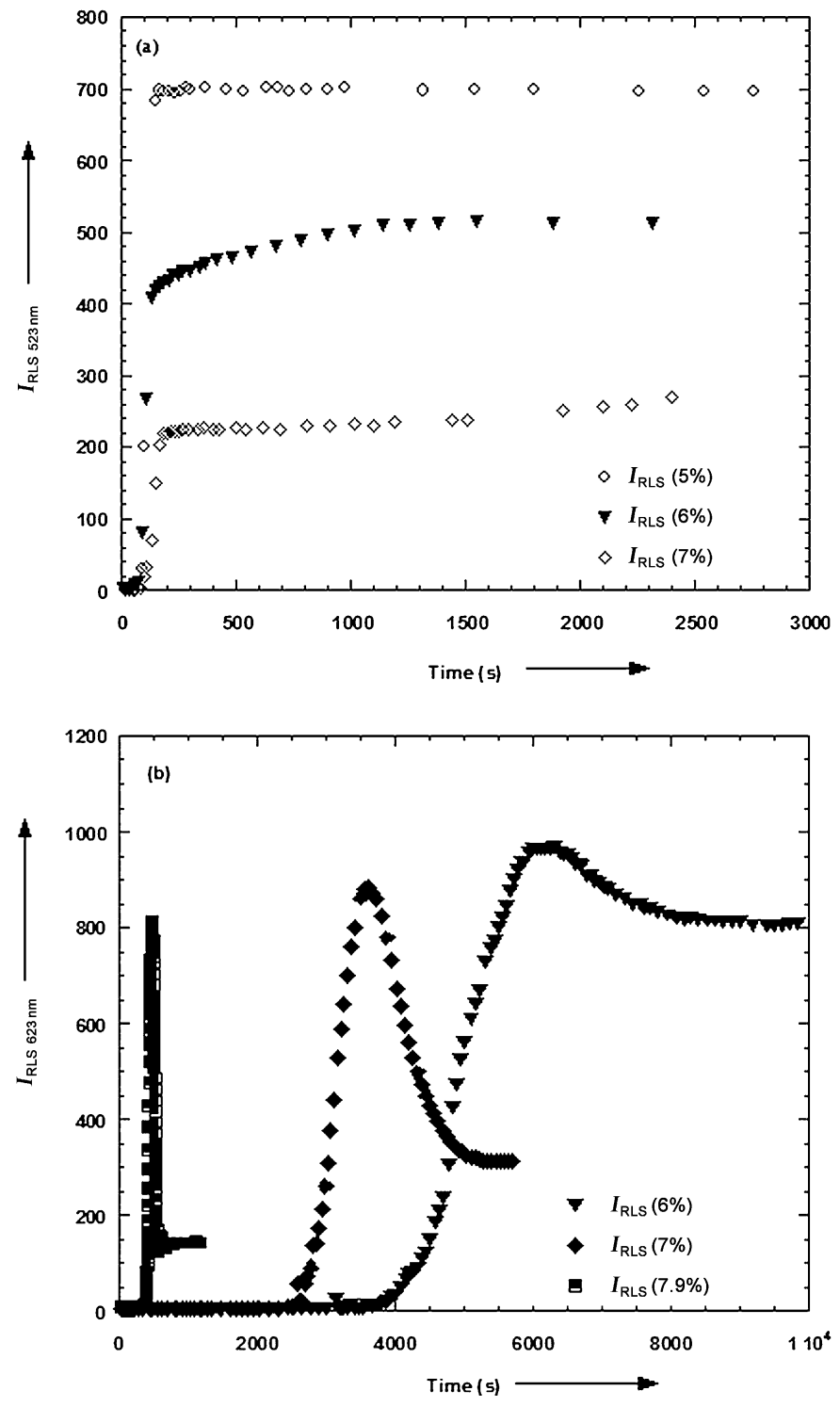

Figure 5. Plots of RLS intensity $\left(I_{\mathrm{RLS}}\right)$ as a function of time for (a) $\mathbf{1} \mathbf{b}$ and (b) $1 \mathrm{c}$ in propanol solution.

Analysis of the plots reported in Figure 5 once again evidences that the different nature of the anion significantly affects formation of the fibrillar network. First, the aggregation process occurs faster in the presence of $\mathbf{1} \mathbf{b}$ than $\mathbf{1} \mathbf{c}$. Of course, because the aggregation rate is affected significantly by the rate at which nucleation occurs, significant differences in the nucleation times were detected for the organogelators considered. Indeed, in the presence of $\mathbf{1} \mathbf{b}$ the nucleation process started immediately, whereas in the presence of $1 \mathrm{c}$ an induction time was observed (from $\approx 300$ to $3700 \mathrm{~s}$ on going from 7.9 to $6.0 \%$, respectively).

On the other hand, kinetic tracks have very different trends. In particular, in the case of $\mathbf{1} \mathbf{b}$, the RLS intensity gradually increases with the time up to a plateau value. By contrast, in the case of $1 \mathrm{c}$, the RLS intensity firstly increases and then decreases to reach a constant value. In the latter case, organogel formation seems to be characterized by the presence of two con- secutive processes. Bearing in mind the hierarchical organization that allows gel-phase formation through a self-assembly process, thereby giving fibers before gel formation, ${ }^{[2]]}$ we presume that the presence of the [2,6-NDS] anion favors previous formation of large aggregates that are subsequently rearranged in smaller and more stable ones present in the gel network. We recently found a similar result by studying the gelation process of geminal imidazolium salts bearing the bromide ion. ${ }^{[11]}$ On the other hand, Terech and co-workers also detected a similar behavior on studying the variation of opacity of organogels formed by 12-hydroxystearic acid, and ascribed it to a contraction of the three-dimensional network proceeding through fiber stacking into bundles. ${ }^{[26]}$

For a better evaluation of the collected data, times and RLS intensities corresponding to the gel $\left(t_{\mathrm{e}}\right.$ and $\left.l_{\mathrm{e}}\right)$ or to the fibrillar intermediate formation $\left(t_{\mathrm{M}}\right.$ and $\left.I_{\mathrm{M}}\right)$ are reported in Table 2.

\begin{tabular}{|c|c|c|c|c|c|}
\hline Organogelator & $\begin{array}{l}\text { Gelator concentration } \\
{[\%, w / w]}\end{array}$ & $\begin{array}{l}t_{\mathrm{M}} \\
{[\mathrm{s}]}\end{array}$ & $I_{\mathrm{M} 523 \mathrm{~nm}}$ & $\begin{array}{l}t_{\mathrm{e}} \\
{[\mathrm{s}]}\end{array}$ & $l_{\mathrm{e} 523 \mathrm{~nm}}$ \\
\hline $1 \mathrm{~b}$ & 5.0 & - & - & 150 & 700 \\
\hline $1 \mathrm{~b}$ & 6.0 & - & - & 130 & 458 \\
\hline $1 \mathrm{~b}$ & 7.0 & - & - & 215 & 225 \\
\hline $1 \mathrm{c}$ & 6.0 & 6180 & 965 & 9400 & 805 \\
\hline $1 \mathrm{c}$ & 7.0 & 3600 & 887 & 5200 & 315 \\
\hline $1 \mathrm{c}$ & 7.9 & 470 & 817 & 550 & 123 \\
\hline
\end{tabular}

On the grounds of $I_{\mathrm{M}}$ values, we may hypothesize that only minor differences in the size of the fibrillar intermediate formed by $1 \mathrm{c}$ occur on increasing the organogelator concentration. Rather, the significant differences detected in the equilibrium values seem to be a consequence of the increasing concentration of free organogelator that induces the rearrangement in the aggregates that constitute the gel phase.

As far as equilibrium times (i.e., the time at which the RLS intensity stays constant) are concerned, a different behavior of the two organogelators can be evidenced. In general, fibrillar network formation occurs faster in the presence of $\mathbf{1} \mathbf{b}$ than $1 \mathrm{c}$. Bearing in mind the results previously obtained for $\Delta H_{\mathrm{f}}$ values, we observed that formation of a more stable network occurs faster. In the presence of $\mathbf{1} \mathbf{b}$, the gelation time does not show significant variations. On the contrary, gelation occurs faster on increasing the concentration of $[p-X y \mid-$ (oim) $\left.)_{2}\right][2,6-N D S]$ in the solution.

The analysis of RLS intensity at the equilibrium shows that in both cases the size of the aggregates gradually decreases with the organogelator concentration. This decrease could be a consequence of the presence of a larger number of fiber nucleation centers, which disfavors the growth of the arms.

To obtain information about the effect that a different solvent might exert on the size of the aggregates in the gel phase, the RLS investigation was also performed in butanol solution. As far as data for $\mathbf{1} \mathbf{b}$ are concerned $(7 \%, w / w)$, gel for- 
mation was also very fast at room temperature (the equilibrium value was reached after $40 \mathrm{~s}$ ). The RLS intensity for the gel state $\left(I_{\mathrm{e}} \approx 140\right)$ was lower than that observed in propanol $\left(I_{\mathrm{e}} \approx 225\right)$, thus revealing the presence of less extensive aggregates. Figure 6 shows plots of RLS intensity as a function of the time corresponding to the gelation process of $1 \mathrm{c}(7 \%$, $\mathrm{w} / \mathrm{w}$ ) in both propanol and butanol solution.

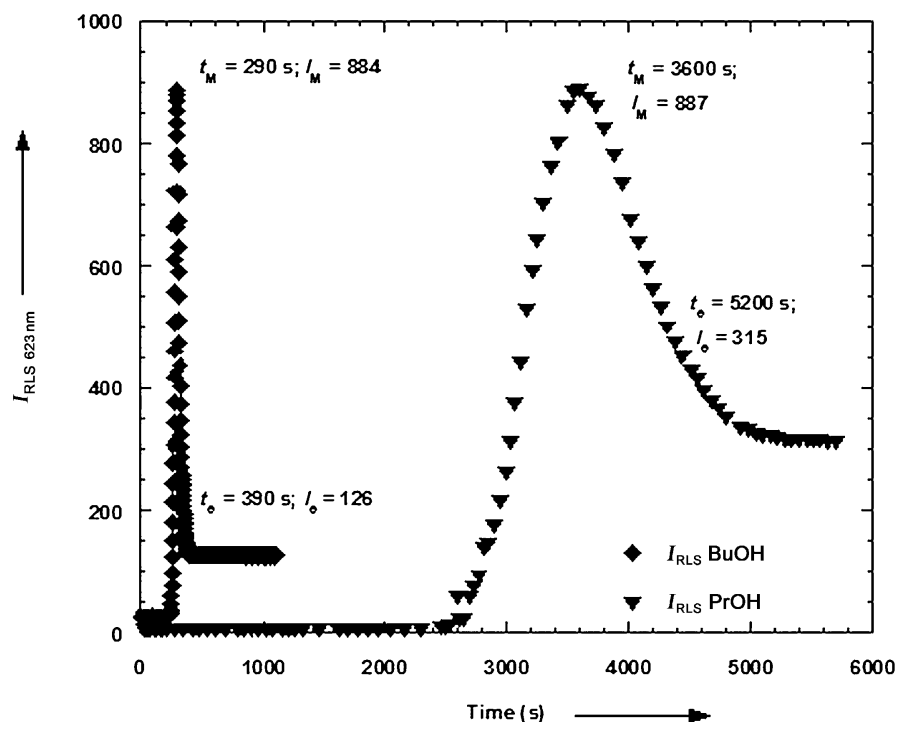

Figure 6. Plots of RLS intensity $\left(I_{\text {RLS }}\right)$ as a function of time for $\mathbf{1} \mathbf{c}$ in propanol $(\nabla)$ and in butanol $(\bullet)$ solution.

In this case the different nature of the solvent does not affect the shape of the kinetic track and the RLS intensity corresponding to the fibrillar intermediate formation. However, it induces a significant decrease both in nucleation and gelation time, and according to what is detected in the presence of $\mathbf{1} \mathbf{b}$, it affects significantly the size of the aggregates characterizing the gel network. Also in this case, on going from propanol to butanol, a significant decrease in the size of aggregates was detected. Finally, the width of the kinetic tracks suggests that significant differences occur in the rate of rearrangement processes on going from the fibrillar intermediate to the gel phase.

\section{SEM measurements}

The morphologies of the obtained gels were also investigated by using SEM measurements. Figure 7 shows SEM images of xerogels prepared from the propanol and butanol gels of both $\mathbf{1} \mathbf{b}$ and $\mathbf{1 c}$ at $7 \%(\mathrm{w} / \mathrm{w})$. Analysis of these images shows that the structure of the anion induces significant differences in the morphologies of the gels. With the percentage of the organogelator the same, the xerogel formed by $\mathbf{1} \mathbf{b}$ seems to have a lamellar organization, whereas that formed by $1 \mathrm{c}$ shows a fibrillar organization. We also evaluated the length of the fibers, and evidenced a higher extension of the fibers corresponding to the xerogel formed by $\mathbf{1} \mathbf{b}$ than that corresponding to the xerogel formed by $\mathbf{1}$ c. Indeed, we found an average length of (a)

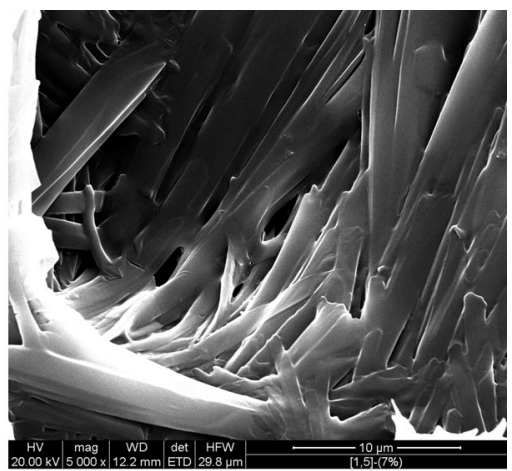

(b)

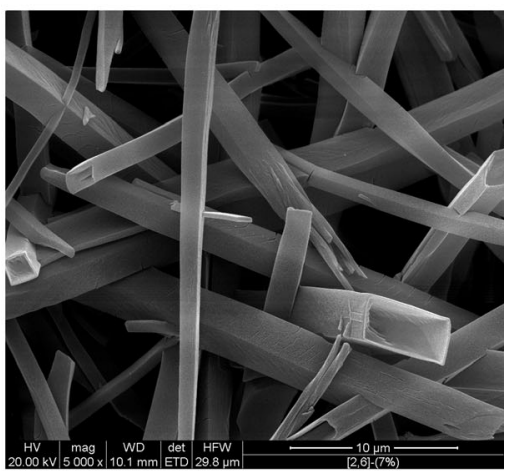

(c)

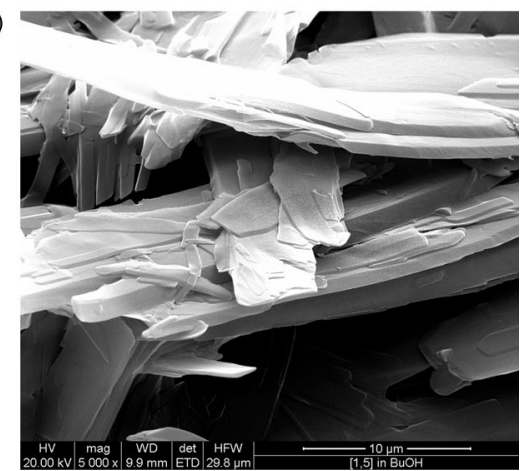

Figure 7. SEM images, at $10 \mu \mathrm{m}$, of xerogels prepared from propanol gels of $\mathbf{1} \mathbf{b}(\mathrm{a})$ and $\mathbf{1} \mathbf{c}(\mathrm{b})$ and from a butanol gel of $\mathbf{1} \mathbf{b}(\mathrm{c})(7 \%, \mathrm{w} / \mathrm{w})$.

$50 \mu \mathrm{m}$ for $\mathbf{1} \mathbf{b}$ and $40 \mu \mathrm{m}$ for $\mathbf{1}$ c. Significant differences were also detected in the fiber width. Indeed, this structural parameter ranges from 0.4 to $2.1 \mu \mathrm{m}$ for the xerogel formed by $1 \mathrm{c}$ and from 0.9 to $2.9 \mu \mathrm{m}$ for the xerogel formed by $\mathbf{1} \mathbf{b}$ (see Figure S2).

Bearing in mind the trend obtained for $T_{\text {gel }}$ values as a function of the organogelator concentration in the case of $\mathbf{1} \mathbf{b}$, and to verify if the above trend could be also ascribed to changes in the morphology of the three-dimensional network, we recorded SEM images corresponding to gel phases formed in propanol at 5.4, 5.6, and 6.0\% (w/w; see Figure S3). Analysis of the SEM images shows that the studied system displays different morphologies as a function of the organogelator concentration. Indeed, at $5.4 \%(\mathrm{w} / \mathrm{w})$ a morphology resembling that of a fibrous structure was detected (Figure S3a,b). However, with the increase in the organogelator concentration, a transition from a fibrous to a lamellar structure can be picked out (Figure S3 c-f). A similar behavior in the relationship of organo- 
gelator concentration and gel morphology was also reported previously by Yi and co-workers. ${ }^{[13 e]}$

To obtain further information about the effects derived from the nature of the solvent, SEM images of the xerogel formed by $\mathbf{1} \mathbf{b}$ obtained from butanol $(7 \%, w / w)$ were also recorded (see Figure 7c). The different nature of the alcohol used did not affect gel morphology. Indeed, in this case a lamellar organization was also observed. However, the network seems to be constituted by wider fibers with respect to those forming the network in propanol, with a width ranging from 3.4 to $6.4 \mu \mathrm{m}$ (see Figure S2).

\section{Conclusion}

The evidence collected by using a combined approach of different methodologies has allowed deeper insights into the role played by gelator structure in determining the gelling abilities of some geminal diimidazolium salts. In particular, analysis of data reported herein sheds light on the contribution derived from the cation or anion structure. For the investigated class of low-molecular-weight gelators, the gelling or nongelling behavior seems to be ruled by the cation structure, as a consequence of the different lengthening of the structure. On the other hand, the overall properties and structural features of the obtained gel phases seem to be determined by the anion properties.

As accounted for by computational investigations and in particular by the results obtained for the cation-anion-cation systems, the 1,3-disubstitution on the benzene central core would give rise to stronger interactions with the anion with respect to 1,4-disubstitution. This, along with an enhanced caging effect of the $\left[\mathrm{m}\right.$-Xyl-(oim) $\left.{ }_{2}{ }^{2+}\right]$ cation, would hamper the establishment of efficient interactions among different ion pairs needed for gel fiber formation. This hypothesis is well supported by thermodynamic dissolution parameters, which indicate for $\left[m\right.$-Xyl-(oim) $\left.{ }_{2}{ }^{2+}\right]$-based salts a higher propensity to interact with solvent molecules and consequently less intense gelator-gelator interactions.

As far as gel phases are concerned, the gelling ability significantly improves on going from mono- to dianions. In the case of naphthalenedisulfonate anions, a simple structural change, such as the use of different positional isomers having different distances between negative centers, induces significant variations in the nature of supramolecular interactions producing the three-dimensional network of the gel. These differences, which are well represented by the fully optimized DFT geometries of the ion pairs $\mathbf{1} \mathbf{b}$ and $\mathbf{1} \mathbf{c}$, would induce significant changes not only in the properties of the obtained soft materials (i.e., thermodynamic stability and morphology), but also in the outcome of the gelation process and in the ability of the gel phase to behave as a self-healing material.

\section{Experimental Section}

\section{Materials}

$\alpha, \alpha^{\prime}$-p-Dibromoxylene, propanol, 2-propanol, ethanol, butanol, pentanol, hexanol, anhydrous acetonitrile, Amberlite IRA-400, Amberlite IR 120 plus, 1,5-naphthalenedisulfonic acid tetrahydrate, 2,6naphthalenedisulfonic acid disodium salt, and $N$-octylimidazole were used as purchased without further purification. Acetone was purified by distillation before use. Methanol was distilled and stored under argon.

\section{General procedure for the synthesis of the dibromide salts $(1 \mathrm{a}, 2 \mathrm{a})^{[12]}$}

$\alpha, \alpha^{\prime}$-Dibromoxylene $(2.48 \mathrm{~g}, 10.8 \mathrm{mmol})$ was dissolved in 2-propanol $(10 \mathrm{~mL})$. The obtained solution was placed in an oil bath at $85^{\circ} \mathrm{C}$. N-Octylimidazole $(3.88 \mathrm{~g}, 21.5 \mathrm{mmol})$ was dissolved in 2propanol $(10 \mathrm{~mL})$ and the solution was added, dropwise, to the $\alpha, \alpha^{\prime}$-dibromoxylene solution. The reaction mixture was heated at $85^{\circ} \mathrm{C}$ for $24 \mathrm{~h}$. After cooling, in the case of $\left.[\mathrm{p} \text {-Xyl-(oim) }]_{2}\right][\mathrm{Br}]_{2}$ the reaction mixture was diluted with acetone $(200 \mathrm{~mL})$ and a white solid was collected by filtration. In the case of $\left[m-X y l-(o i m)_{2}\right][B r]_{2}$ after concentration under reduced pressure, a yellow viscous oil was obtained, which was washed several times with diethyl ether. The obtained dibromide salts were dissolved in anhydrous methanol and stirred overnight $(12 \mathrm{~h})$ at room temperature, in the presence of active charcoal ( $1 \%$ by weight). After filtration on neutral aluminum oxide and concentration in vacuo, the desired salt was obtained.

3,3'-Di- $n$-octyl-1, 1'-(1,4-phenylenedimethylene)diimidazolium dibromide (1 a): White solid; m.p. $150-152^{\circ} \mathrm{C}$; yield $82 \%$; ${ }^{1} \mathrm{H}$ NMR (300 MHz, [D 6 DMSO): $\delta=9.40(\mathrm{~s}, 2 \mathrm{H}), 7.82(\mathrm{~d}, J=1.5 \mathrm{~Hz}, 4 \mathrm{H}), 7.47$ $(\mathrm{s}, 4 \mathrm{H}), 5.44(\mathrm{~s}, 4 \mathrm{H}), 4.16(\mathrm{t}, J=7.3 \mathrm{~Hz}, 4 \mathrm{H}), 1.79(\mathrm{~m}, 4 \mathrm{H}), 1.19(\mathrm{~m}$, $20 \mathrm{H}), 0.86 \mathrm{ppm}(\mathrm{t}, J=6.6 \mathrm{~Hz}, 6 \mathrm{H}) ;{ }^{13} \mathrm{C}$ NMR $\left(250 \mathrm{MHz},\left[\mathrm{D}_{6}\right] \mathrm{DMSO}\right)$ : $\delta=136.3,135.6,129.0,123.0,122.7,51.6,49.1,31.2,29.4,28.6,28.4$, 25.6, 22.2, $14.1 \mathrm{ppm}$; elemental analysis calcd for $\mathrm{C}_{30} \mathrm{H}_{48} \mathrm{Br}_{2} \mathrm{~N}_{4}$ : C 57.67, H 7.75, Br 25.59, N 8.97; found: C 57.70, H 7.60, Br 25.49, N 9.08 .

3,3'-Di-n-octyl-1,1'-(1,3-phenylenedimethylene)diimidazolium dibromide (2a): Yellow viscous oil; yield $86 \%$; ${ }^{1} \mathrm{H} N M R \quad(300 \mathrm{MHz}$, [D6]DMSO): $\delta=9.44(\mathrm{~s}, 2 \mathrm{H}), 7.83$ (dd, $J=1.2,4.2 \mathrm{~Hz}, 4 \mathrm{H}$ ), 7.59 (s, $1 \mathrm{H}), 7.43(\mathrm{~m}, 3 \mathrm{H}), 5.45(\mathrm{~s}, 4 \mathrm{H}), 4.18(\mathrm{t}, J=7.2 \mathrm{~Hz}, 4 \mathrm{H}), 1.79$ (qt, $J=$ $6.7 \mathrm{~Hz}, 4 \mathrm{H}), 1.23(\mathrm{~m}, 20 \mathrm{H}), 0.86 \mathrm{ppm}(\mathrm{t}, J=6.6 \mathrm{~Hz}, 6 \mathrm{H}) ;{ }^{13} \mathrm{C}$ NMR (250 MHz, [D6]DMSO): $\delta=137.1,136.5,130.6,129.4,129.3,123.7$, $123.4,52.5,49.9,32.0,30.1,29.3,29.2,26.4,22.9,14.8$ ppm; elemental analysis calcd for $\mathrm{C}_{30} \mathrm{H}_{48} \mathrm{Br}_{2} \mathrm{~N}_{4}$ : C 57.69, H 7.75, $\mathrm{Br} 25.59, \mathrm{~N}$ 8.97; found: C 57.61, H 7.79, Br 25.74, N 8.71.

\section{General procedure for the synthesis of the naphthalenedi- sulfonate salts $(1 \mathrm{~b}, \mathrm{c} \text { and } 2 \mathrm{~b}, \mathrm{c})^{[27]}$}

A column packed with anion-exchange Amberlite resin IRA-400 (chloride form, $11.50 \mathrm{~g}$ ) was used. To convert the chloride form of the resin into the hydroxide form, it was first washed with an aqueous solution of $\mathrm{NaOH}(570 \mathrm{~mL}, 10 \% \mathrm{w} / \mathrm{v})$ and subsequently with water until the eluate was neutral. A binary mixture of methanol/ water $(70: 30, \mathrm{v} / \mathrm{v})$ was used as eluent. Bromide salt $(1.68 \mathrm{~g}$, $2.69 \mathrm{mmol}$ ) was dissolved in the binary mixture $(50 \mathrm{~mL})$ and eluted. The eluate was collected in a flask containing a solution of the naphthalenedisulfonic acid in stoichiometric amount. The neutral solution was concentrated in vacuo and the residue was crystal- 
lized from ethanol. In the case of $\mathbf{2} \mathbf{b}$ and $\mathbf{2} \mathbf{c}$ the salts were dissolved in ethanol and solutions were heated in the presence of active charcoal. The hot solution was filtered and the solvent was removed under vacuum. The 2,6-naphthalenedisulfonic acid was obtained by elution of a water solution of the corresponding sodium salt through an Amberlite IR 120 plus column. In both cases, the silver nitrate test, performed to verify the presence of residual bromide anion, gave a negative result.

3,3'-Di-n-octyl-1,1'-(1,4-phenylenedimethylene)diimidazolium 1,5naphthalenedisulfonate $(\mathbf{1} \mathbf{b})$ : White solid; m.p. $198-204^{\circ} \mathrm{C}$; yield $95 \%$; ${ }^{1} \mathrm{H}$ NMR (300 MHz, [D $\mathrm{D}_{6}$ DMSO): $\delta=9.31(\mathrm{~s}, 2 \mathrm{H}), 8.87(\mathrm{~d}, J=$ $8.4 \mathrm{~Hz}, 2 \mathrm{H}), 7.92(\mathrm{~d}, J=6.9 \mathrm{~Hz}, 2 \mathrm{H}), 7.76(\mathrm{~m}, 4 \mathrm{H}), 7.37(\mathrm{~m}, 6 \mathrm{H}), 5.37$ $(\mathrm{s}, 4 \mathrm{H}), 4.13(\mathrm{t}, J=7.2 \mathrm{~Hz}, 4 \mathrm{H}), 1.76(\mathrm{~m}, 4 \mathrm{H}), 1.23(\mathrm{~m}, 20 \mathrm{H})$, $0.84 \mathrm{ppm}(\mathrm{t}, J=6.6 \mathrm{~Hz}, 6 \mathrm{H}) ;{ }^{13} \mathrm{C} \mathrm{NMR}\left(250 \mathrm{MHz},\left[\mathrm{D}_{6}\right] \mathrm{DMSO}\right): \delta=$ $144.1,136.3,135.5,129.7,129.2,128.9,124.1,123.9,123.0,122.6$, 51.6, 49.1, 31.2, 29.4, 28.6, 28.4, 25.6, 22.2, 14.1 ppm; elemental analysis calcd for $\mathrm{C}_{40} \mathrm{H}_{54} \mathrm{~N}_{4} \mathrm{O}_{6} \mathrm{~S}_{2}: \mathrm{C} 63.97, \mathrm{H} 7.25, \mathrm{~N} 7.46, \mathrm{~S} 8.52$; found: C 63.80, H 7.30, N 7.54, S 8.48.

3,3'-Di-n-octyl-1,1'-(1,4-phenylenedimethylene)diimidazolium 2,6naphthalenedisulfonate (1 c): White solid; m.p. $228-231^{\circ} \mathrm{C}$; yield $92 \%$; ${ }^{1} \mathrm{H}$ NMR $\left(300 \mathrm{MHz},\left[\mathrm{D}_{6}\right] \mathrm{DMSO}\right): \delta=9.32(\mathrm{~s}, 2 \mathrm{H}), 8.11(\mathrm{~s}, 2 \mathrm{H})$, $7.87(\mathrm{~d}, J=8.7 \mathrm{~Hz}, 2 \mathrm{H}), 7.79(\mathrm{~m}, 4 \mathrm{H}), 7.70(\mathrm{dd}, J=1.2,8.4 \mathrm{~Hz}, 2 \mathrm{H})$, $7.39(\mathrm{~s}, 4 \mathrm{H}), 5.39(\mathrm{~s}, 4 \mathrm{H}), 4.14(\mathrm{t}, J=7.2 \mathrm{~Hz}, 4 \mathrm{H}), 1.76(\mathrm{~m}, J=6.6 \mathrm{~Hz}$, $4 \mathrm{H}), 1.25(\mathrm{~m}, 20 \mathrm{H}), 0.84 \mathrm{ppm}(\mathrm{t}, J=6.7 \mathrm{~Hz}, 6 \mathrm{H}) ;{ }^{13} \mathrm{C} \mathrm{NMR}(250 \mathrm{MHz}$, [D $\mathrm{D}_{6}$ ]DMSO): $\delta=146.2,136.4,135.5,132.1,128.9,128.1,124.4,123.9$, 123.1, 122.7, 51.6, 49.2, 31.3, 29.4, 28.6, 28.4, 25.7, 22.2, 14.1 ppm; elemental analysis calcd for $\mathrm{C}_{40} \mathrm{H}_{54} \mathrm{~N}_{4} \mathrm{O}_{6} \mathrm{~S}_{2}: \mathrm{C} 63.97, \mathrm{H} 7.25, \mathrm{~N} 7.46, \mathrm{~S}$ 8.52; found: C 63.87, H 7.12, N 7.38, S 8.60.

3,3'-Di-n-octyl-1,1'-(1,3-phenylenedimethylene)diimidazolium 1,5naphthalenedisulfonate (2 b): Colorless oil; yield $96 \%$; ${ }^{1} \mathrm{H}$ NMR (300 MHz, [D6]DMSO): $\delta=9.35$ (s, 2H), 8.9 (d, $J=8.5 \mathrm{~Hz}, 2 \mathrm{H}), 7.93$ $(\mathrm{dd}, J=1.1,7.2 \mathrm{~Hz}, 2 \mathrm{H}), 7.78(\mathrm{~m}, 4 \mathrm{H}), 7.51(\mathrm{~s}, 1 \mathrm{H}), 7.40(\mathrm{~m}, 6 \mathrm{H})$, $5.38(\mathrm{~s}, 4 \mathrm{H}), 4.13(\mathrm{t}, J=7.3 \mathrm{~Hz}, 4 \mathrm{H}), 1.76(\mathrm{~m}, 4 \mathrm{H}), 1.23(\mathrm{~m}, 20 \mathrm{H})$, $0.87 \mathrm{ppm}(\mathrm{t}, J=6.3 \mathrm{~Hz}, 6 \mathrm{H}) ;{ }^{13} \mathrm{C}$ NMR $(250 \mathrm{MHz},[\mathrm{D} 6] \mathrm{DMSO}) \delta=$ $144.1,136.4,135.7,129.9,129.7,129.2,128.7,128.7,124.2,124.0$, 123.0, 122.7, 51.8, 49.2, 31.3, 29.4, 28.6, 28.5, 25.7, 22.2, 14.1 ppm; elemental analysis calcd for $\mathrm{C}_{40} \mathrm{H}_{54} \mathrm{~N}_{4} \mathrm{O}_{6} \mathrm{~S}_{2}: \mathrm{C} 53.40, \mathrm{H} 7.08, \mathrm{~N} 7.47, \mathrm{~S}$ 8.54; found: C 53.45, H 7.12, N 7.41, S 8.46.

3,3'-Di-n-octyl-1,1'-(1,3-phenylenedimethylene)diimidazolium 2,6naphthalenedisulfonate (2c): Yellow oil; yield 97\%; ${ }^{1} \mathrm{H}$ NMR (300 MHz, [D6]DMSO): $\delta=9.38(\mathrm{~s}, 2 \mathrm{H}), 8.17(\mathrm{~s}, 2 \mathrm{H}), 7.95(\mathrm{~d}, J=$ $8.7 \mathrm{~Hz}, 2 \mathrm{H}), 7.86(\mathrm{~m}, 4 \mathrm{H}), 7.76(\mathrm{~d}, J=8.4 \mathrm{~Hz}, 2 \mathrm{H}), 7.60(\mathrm{~s}, 1 \mathrm{H}), 7.42$ $(\mathrm{s}, 3 \mathrm{H}), 5.47(\mathrm{~s}, 4 \mathrm{H}), 4.20(\mathrm{t}, J=7.2 \mathrm{~Hz}, 4 \mathrm{H}), 1.82(\mathrm{~m}, 4 \mathrm{H}), 1.28(\mathrm{~m}$, $20 \mathrm{H}), 0.91 \mathrm{ppm}(\mathrm{t}, J=4.2 \mathrm{~Hz}, 6 \mathrm{H}) ;{ }^{13} \mathrm{C}$ NMR $(250 \mathrm{MHz}$, [D6]DMSO): $\delta=146.2,136.4,135.7,132.1,129.9,128.7,128.7,128.2,124.4$, 123.9, 123.0, 122.7, 51.8, 49.12, 31.3, 29.4, 28.6, 28.5, 25.7, 22.2, $14.1 \mathrm{ppm}$; elemental analysis calcd for $\mathrm{C}_{40} \mathrm{H}_{54} \mathrm{~N}_{4} \mathrm{O}_{6} \mathrm{~S}_{2}: \mathrm{C} 53.40, \mathrm{H}$ 7.08, N 7.47, S 8.54; found: C 53.33, H 7.01, N 7.45, S 8.67.

\section{Preparation of gels and determination of $T_{\text {gel }}$}

Gels were prepared by weighing into a screw-capped sample vial (diameter $1 \mathrm{~cm})$ the amount of salt and solvent $(\approx 250 \mathrm{mg})$. For the investigated concentration range, samples different by $0.5 \%(\mathrm{w} / \mathrm{w})$ were tested. The sample vial was heated in an oil bath until a clear solution was obtained. The vial was then cooled and stored at $4{ }^{\circ} \mathrm{C}$ overnight $(12 \mathrm{~h})$. The tube inversion test method was used to examine gel formation in different solvents. This test was performed at $293 \mathrm{~K}$. To determine the $T_{\text {gel }}$ values, a lead ball (weighing less than $100 \mathrm{mg}$ ) was placed on top of the gel and the vial was placed in a water bath. The bath temperature was gradu- ally increased until the gel melted $\left(T_{\text {gel }}\right)$ and the lead ball started to move downward. ${ }^{[28]} T_{\text {gel }}$ values were reproducible within $1 \mathrm{~K}$.

\section{Determination of $\Delta H_{\text {diss }}$ and $\Delta S_{\text {diss }}$}

The salt $(5 \mathrm{mg})$ was weighed into a screw-capped vial and the desired solvent $(1 \mathrm{~mL})$ was added. The vial was left for $24 \mathrm{~h}$ without stirring, in a thermostatic bath preset at the required temperature. After this time an aliquot $(100 \mu \mathrm{L})$ of the supernatant solution was withdrawn and diluted to $1 \mathrm{~mL}$. The UV/Vis spectra of the obtained solution were recorded with a Beckman Coulter DU 800 spectrometer. The working wavelength was chosen as the one corresponding to the maximum absorbance value of the obtained spectrum. The logarithms of the dissolved mole fraction values were plotted versus $1 / T$ and fitted by means of the van't Hoff equation ${ }^{[16]}$ to obtain $\Delta H_{\text {diss }}$ and $\Delta S_{\text {diss }}$ values.

\section{Computational details}

Geometry optimizations were performed in the DFT framework by using the new hybrid meta exchange-correlation functional M062X of Truhlar and Zhao, ${ }^{[29]}$ recommended for the accurate description of noncovalent interactions in main-group compounds. Dunning's correlation-consistent polarized valence double zeta, cc-pvdz, ${ }^{[30]}$ was used as basis set. The nature of minima of the potential energy surface for all the systems investigated herein was checked by inspection of the calculated vibrational harmonic frequencies. The energy values were corrected for the zero-point vibrational contribution. Atom-centered charges were evaluated by fitting them to the molecular electrostatic potential calculated at the Moeller-Plesset second-order perturbation theory level on the DFT optimized geometries. All calculations were performed by using the Gaussian 09 package. ${ }^{[31]}$

\section{RLS measurements}

RLS measurements were performed on a spectrofluorophotometer (Jasco FP-777W) by using a synchronous scanning mode in which the emission and excitation monochromators were preset to identical wavelengths. The RLS spectrum was recorded from 300 to $600 \mathrm{~nm}$ with both the excitation and emission slit widths set at $1.5 \mathrm{~nm}$. The working wavelength was chosen as the one corresponding to the intensity maximum of the obtained spectrum. Samples for a typical kinetic measurement were prepared by injecting in a quartz cuvette (light path $0.2 \mathrm{~cm}$ ) the limpid hot solution of salt. Spectra were recorded until gel formation. The gel phase obtained at the end of the measurement was stable after the tube inversion test.

\section{Thixotropy tests}

A magnetic stirring bar (length $8 \mathrm{~mm}$, height $3 \mathrm{~mm}$ ) was added to a preformed gel in a screw-capped vial. The sample was stirred for $5 \mathrm{~min}$ at $1000 \mathrm{rpm}$ and left to stand at room temperature for $12 \mathrm{~h}$. The stability of the obtained phase was investigated by the tube inversion test.

\section{Sonotropic tests}

A gel, preformed in a screw-capped vial, was placed in an ultrasonic cleaning bath and irradiated for $5 \mathrm{~min}$ at the frequency of $45 \mathrm{kHz}$ and with a power of $200 \mathrm{~W}$. The suspension obtained was 
left to stand for $12 \mathrm{~h}$. After this time the stability of the obtained material was tested by means of the tube inversion test.

\section{Scanning electron microscopy}

SEM images were recorded by using an FEI Quanta 200 instrument with $20 \mathrm{kV}$ operating voltage. The gel was placed on the stub and the solvent was evaporated under vacuum to form a xerogel. The dry sample thus obtained was shielded by gold.

\section{Acknowledgements}

We thank the University of Palermo and MIUR (FIRB 2010RBFR10BF5V) for financial support.

Keywords: gels • imidazolium salts · isomers · self-assembly · substituent effects

[1] a) D. K. Smith, Chem. Commun. 2006, 34-44; b) J. W. Goodby, I. M. Saez S. J. Cowling, V. Görtz, M. Draper, A. W. Hall, S. Sia, G. Cosquer, S. E. Lee, E. P. Raynes, Angew. Chem. 2008, 120, 2794-2828; Angew. Chem. Int. Ed. 2008, 47, 2754-2787; c) H. K. Bisoyi, S. Kumar, Chem. Soc. Rev. 2011, 40, 306-319; d) D. J. A. Cameron, M. P. Shaver, Chem. Soc. Rev. 2011, 40, 1761-1776; e) L. Zhao, Z. Lin, Soft Matter 2011, 7, 10520-10535; f) C. Fong, T. Le, C. J. Drummond, Chem. Soc. Rev. 2012, 41, 1297-1322; g) M. Seo, J. Park, S. Y. Kim, Org. Biomol. Chem. 2012, 10, 5332-5342.

[2] a) A. R. Hirst, D. K. Smith, Chem. Eur. J. 2005, 11, 5496-5508; b) N. M. Sangeetha, U. Maitra, Chem. Soc. Rev. 2005, 34, 821-836; c) X. Huang, S. R. Raghavan, P. Terech, R. G. Weiss, J. Am. Chem. Soc. 2006, 128, 15341 -15352; d) G. John, P. K. Vemula, Soft Matter 2006, 2, 909-914 e) G. Roy, J. F. Miravet, B. Escuder, C. Sanchez, M. Llusar, J. Mater. Chem. 2006, 16, 1817-1824; f) A. R. Hirst, B. Escuder, J. F. Miravet, D. K. Smith Angew. Chem. 2008, 120, 8122-8139; Angew. Chem. Int. Ed. 2008, 47, $8002-8018$; g) S. Iqbal, J. F. Miravet, B. Escuder, Eur. J. Org. Chem. 2008, 4580-4590; h) G. Cravotto, P. Cintas, Chem. Soc. Rev. 2009, 38, 2684 2697 ; i) S. Banerjee, R. K. Das, U. Maitra, J. Mater. Chem. 2009, 19, 66496687 ; j) B. Escuder, F. Rodriguez-Llansola, J. F. Miravet, New J. Chem. 2010, 34, 1044-1054; k) J.-L. Li, X.-Y. Liu, Adv. Funct. Mater. 2010, 20 3196-3216; I) Y. Suzaki, T. Taira, K. Osakada, J. Mater. Chem. 2011, 21, $930-938$.

[3] a) D. J. Abdallah, L. Lu, R. G. Weiss, Chem. Mater. 1999, 11, 2907-2911; b) P. Terech, E. Ostuni, R. G. Weiss, J. Phys. Chem. 1996, 100, 3759-3766.

[4] a) K. Sada, T. Tani, S. Shinkai, Synlett 2006, 2364-2374; b) M. Suzuki, K. Hanabusa, Chem. Soc. Rev. 2010, 39, 455-463.

[5] P. Terech, R. G. Weiss, Chem. Rev. 1997, 97, 3133-3160.

[6] P. Dastidar, Chem. Soc. Rev. 2008, 37, 2699-2715.

[7] a) J. Y. Kim, T. H. Kim, D. Y. Kim, N.-G. Park, K.-D. Ahn, J. Power Sources 2008, 175, 692-697; b) J. Casamada Ribot, C. Guerrero-Sanchez, R. Hoogenboom, U. S. Schubert, J. Mater. Chem. 2010, 20, 8279-8294; c) J. Casamada Ribot, C. Guerrero-Sanchez, R. Hoogenboom, U. S. Schubert, Chem. Commun. 2010, 46, 6971-6973.

[8] L. Viau, C. Tourné-Péteilh, J. M. Devoiselle, A. Vioux, Chem. Commun. 2010, 46, 228-230.

[9] M. Cai, Y. Liang, F. Zhou, W. Liu, J. Mater. Chem. 2011, 21, 13399-13405.

[10] U. K. Das, D. R. Trivedi, N. N. Adarsh, P. Dastidar, J. Org. Chem. 2009, 74, $7111-7121$.
[11] F. D'Anna, P. Vitale, S. Marullo, R. Noto, Langmuir 2012, 28, 10849 10859.

[12] F. D'Anna, F. Ferrante, R. Noto, Chem. Eur. J. 2009, 15, 13059-13068.

[13] a) K. J. C. van Bommel, A. Friggeri, S. Shinkai, Angew. Chem. 2003, 115, 1010-1030; Angew. Chem. Int. Ed. 2003, 42, 980-999; b) L. A. Estroff, A. D. Hamilton, Chem. Rev. 2004, 104, 1201-1218; c) J. M. Lehn, Chem. Soc. Rev. 2007, 36, $151-160$; d) P. Cordier, F. Tournilhac, C. Soulié-Ziakovic, L. Leibler, Nature 2008, 451, 977-980; e) X. Yu, Q. Liu, J. Wu, M. Zhang, X. Cao, S. Zhang, Q. Wang, L. Chen, T. Yi, Chem. Eur. J. 2010, 16, 9099-9106.

[14] a) T. Lindahl, Nature 1993, 362, 709-715; b) R. Han, K. P. Campbell, Curr. Opin. Cell Biol. 2007, 19, 409-416; c) D. Taylor, J. Mater. Sci. 2007, 42, $8911-8918$; d) M. W. Urban, Handbook of Stimuli Responsive Materials, Wiley-VCH, Weinheim, 2011.

[15] a) D. J. Abdallah, R. G. Weiss, Adv. Mater. 2000, 12, 1237-1247; b) A. R. Hirst, D. K. Smith, K. P. Harrington, Chem. Eur. J. 2005, 11, 6552-6559; c) S. Yagai, T. Nakajima, K. Kishikawa, S. Kohmoto, T. Karatsu, A. Kitamura, J. Am. Chem. Soc. 2005, 127, 11134-11139.

[16] a) J. W. Mullin, Solution and Solubility. Crystallization, Butterworth-Heinemann, Oxford, 1992, pp. 81-127; b) D. H. M. Beiny, J. W. Mullin, J. Chem. Eng. Data 1987, 32, 9-10.

[17] S. R. Rogharan, B. H. Cipriano in Gel Formation: Phase Diagrams using Tabletop Rheology and Calorimetry in Molecular Gels, Materials with Self-Assembled Fibrillary Networks (Eds.: R. G. Weiss, P. Terech), Springer, Dordrecht, 2006.

[18] D. J. Adams, K. Morris, L. Chen, L. C. Serpell, J. Bacsa, G. M. Day, Soft Matter 2010, 6, 4144-4156.

[19] a) O. Gronwald, K. Sakurai, R. Luboradzki, T. Kimura, S. Shinkai, Carbohydr. Res. 2001, 331, 307-318; b) J. Chen, J. W. Kampf, A. J. McNeil, Langmuir 2010, 26, 13076-13080; c) M. L. Muro-Small, J. Chen, A. J. McNeil, Langmuir 2011, 27, 13248-13253.

[20] A. R. Hirst, I. A. Coates, T. R. Biucheteau, J. F. Miravet, B. Escuder, V. Castelletto, I. W. Hamley, D. K. Smith, J. Am. Chem. Soc. 2008, 130, $9113-$ 9121.

[21] C. Reichardt, Solvents and Solvent Effects in Organic Chemistry, WileyVCH, Weinheim, 1988, pp. 366-371.

[22] a) M. Raynal, L. Boutellier, Chem. Commun. 2011, 47, 8271-8273; b) P. Curcio, F. Allix, G. Pickaert, B. Jamart-Grégoire, Chem. Eur. J. 2011, 17, 13603-13612; c) J. Kaszynska, A. Łapiński, R. Luboradzki, J. Tritt-Goc, Tetrahedron 2012, 68, 3803-3810.

[23] K. Murata, M. Aoki, T. Sizuki, T. Harado, H. Kawabata, T. Komori, F. Ohseto, K. Ueda, S. Shinkai, J. Am. Chem. Soc. 1994, 116, 6664.

[24] a) H. Zhang, K. Li, H. Liang, J. Wang, Colloids Surf. A 2008, 329, 75-81; b) J. Zheng, X. Wu, M. Wang, D. Ran, W. Xu, J. Yang, Talanta 2008, 74, 526-532; c) J.-J. Wu, N. Li, K.-A. Li, F. Liu, J. Phys. Chem. B 2008, 112, $8134-8138$.

[25] a) J. Anglister, I. Z. Steinberg, Chem. Phys. Lett. 1979, 65, 50-54; b) J. Anglister, I. Z. Steinberg, J. Chem. Phys. 1983, 78, 5358-5368.

[26] P. Terech, D. Pasquier, V. Bordas, C. Rossat, Langmuir 2000, 16, 4485 4494.

[27] I. Dinarès, C. Garcia de Miguel, A. Ibáñez, N. Mesquida, E. Alcalde, Green Chem. 2009, 11, 1507-1510.

[28] A. Takahashi, M. Sakai, T. Kato, Polym. J. 1980, 12, 335-341.

[29] Y. Zhao, D. G. Truhlar, Theor. Chem. Acc. 2008, 120, 215-241.

[30] T. H. Dunning, J. Chem. Phys. 1989, 90, 1007-1023.

[31] M. J. Frisch, Gaussian 09, Revision C.01 Gaussian, Inc., Wallingford CT, 2009. See full citation in the Supporting Information.

Received: January 19, 2013

Published online on March 1, 2013 